\title{
PRIMEROS PASOS EN LA POSTUROGRAFÍA DINÁMICA COMPUTARIZADA
}

\section{First steps in the Computarized Dynamic Posturography}

\author{
Cristina CORDERO-CIVANTOS; María Isabel CALLE-CABANILLAS
}

SACYL. Hospital Universitario de Burgos. Servicio de Otorrinolaringología y patología Cervicofacial. Burgos. España.

Correspondencia: cris.corciv@gmail.com

Fecha de recepción: 3 de diciembre de 2017

Fecha de aceptación: 11 de diciembre de 2017

Fecha de publicación: 14 de diciembre de 2017

Fecha de publicación del fascículo: 1 de septiembre de 2018

Conflicto de intereses: Los autores declaran no tener conflictos de intereses

Imágenes: Los autores declaran haber obtenido las imágenes con el permiso de los pacientes

Política de derechos y autoarchivo: se permite el autoarchivo de la versión post-print (SHERPA/RoMEO)

Licencia CC BY-NC-ND. Licencia Creative Commons Atribución-NoComercial-SinDerivar 4.0 Internacional

Universidad de Salamanca. Su comercialización está sujeta al permiso del editor

\begin{abstract}
RESUMEN Introducción y objetivo: La posturografía dinámica computarizada (CDP) es una técnica que nos va a permitir cuantificar de forma objetiva, como las diferentes patologías afectan la estabilidad postural de los pacientes y su evolución. Metodología: revisión narrativa. Resultados: En este trabajo vamos a repasar como se realizan y analizan las diferentes pruebas de la CDP; y su utilidad diagnóstica y terapéutica en la práctica otorrinolaringológica. Discusión y conclusiones: La posturografía dinámica computarizada es una prueba diagnóstico-terapéutica que permite cuantificar de forma objetiva y reproducible la estabilidad del paciente. Y aporta información relevante para planificar el tratamiento rehabilitador, así como para monitorizar su evolución.
\end{abstract}

PALABRAS CLAVE posturografía dinámica computarizada; CDP; inestabilidad; centro de gravedad; límites de estabilidad, estrategia de equilibrio; patrón afisiológico

SUMMARY Introduction and objective: The Computarized Dynamic Posturography (CDP) is a technique that will allow us to quantify in an objective way, how the different diseases affect patients postural stability and its evolution. Method: Narrative revision. Results: In this paper we will review how the different CDP tests are performed and analyzed; and its diagnostic-therapeutic utility in otorhinolaryngological practice. Discussion and conclusion: The Computarized Dynamic Posturography is a diagnostic and therapeutic test that allows to quantify objectively and reproducible patient stability. And provides relevant information for planning the rehabilitation treatment, as well as to monitor its evolution.

KEYWORDS $\quad$ computarized dynamic posturography; CDP; inestability; gravity center; stability limits; balance strategy; aphysiological pattern 


\section{INTRODUCCIÓN}

La posturografía dinámica computarizada (en inglés Computarized Dynamic Posturography, CDP) es una técnica para la valoración objetiva de la estabilidad postural en bipedestación [1], que se puede definir como el control del centro de masas con respecto a la base de soporte, tanto en situaciones estáticas como dinámicas.

El control postural es una función básica para el organismo, que le permite desarrollar sus actividades de la vida cotidiana y que se consigue a través de la integración en el sistema nervioso central de la información somatosensorial, visual y vestibular. La importancia relativa de cada una de esas aferencias para el mantenimiento del equilibrio es lo que determinaremos con la CDP [2].

Para entender los fundamentos de la CDP, es preciso definir el concepto de centro de masas, siendo el punto alrededor del cual la masa del cuerpo se distribuye de manera homogénea, que en ocasiones se denomina centro de gravedad y es el punto a través del cual actúa la fuerza de la gravedad (localizado teóricamente a la altura del ombligo). El cuerpo ejerce una fuerza sobre el suelo, en oposición a la fuerza de la gravedad y el punto de aplicación de esa fuerza es el centro de presiones. Son 2 conceptos relacionados, «en un modelo postural que considera las oscilaciones del sujeto similares a las que hace un péndulo en posición invertida, la segunda derivada del desplazamiento horizontal del centro de masas es proporcional al componente horizontal de la fuerza de reacción que ejerce el apoyo sobre el suelo» [3], así a partir de la fuerza realizada sobre la plataforma se puede obtener el valor del centro de presiones, que se utiliza como equivalente al centro de masas, que no es fácil de medir.

La CDP se basa en la detección del desplazamiento del centro de presiones corporal ante diferentes situaciones de conflicto sensorial. Las oscilaciones registradas se comparan con las de un grupo de sujetos normales de su misma edad, sexo y altura; de tal forma que menores oscilaciones significan mayor estabilidad, estando el valor máximo representado por el $100 \%$ y viceversa, siendo la caída el $0 \%$. El equipo del que disponemos, Smart Balance Master desarrollado por Neurocom ${ }^{\circledR}$ (Figura 1), consta de un soporte informático, una plataforma dinamométrica móvil y un entorno visual, que pueden permanecer fijos 0 desplazarse proporcionalmente al movimiento que experimenta el paciente durante la exploración (oscilaciones referenciadas a las corporales). La superficie de la plataforma contacta con cuatro transductores de presión simétricamente situados, que miden las fuerzas verticales $y$ un transductor central que mide las fuerzas horizontales a lo largo del eje anteroposterior en el plano paralelo al suelo [4].

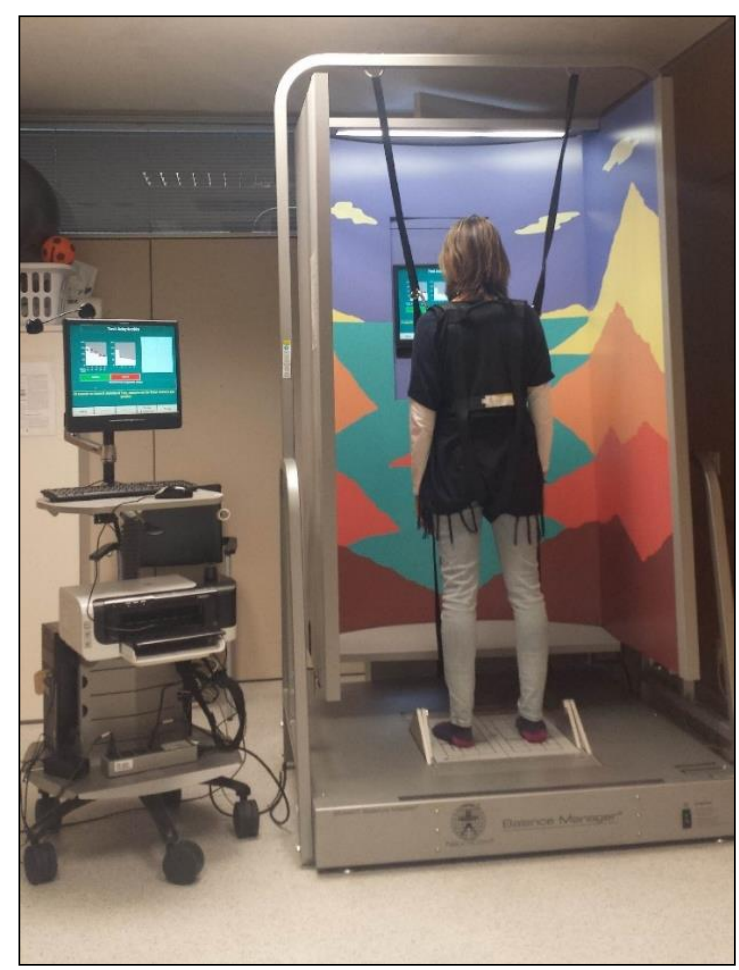

Figura 1. Posturógrafo. Neurocom Smart Balance Master®. Paciente posicionado para realizar el estudio.

La información obtenida mediante la CDP complementa a las insustituibles anamnesis y exploración física, documentando de forma objetiva las alteraciones del control postural, así como aquellos patrones afisiológicos sugerentes de una exageración de la sintomatología [5].

$\mathrm{Y}$ aunque no es cierto que sea considerada como gold standard, ya que ninguna prueba vestibular cumple los criterios de la definición clásica en medicina para tal consideración, la CDP valora el control postural de forma análoga, a las baterías de pruebas audiométricas la audición [6]. 
Con la CDP se pueden realizar las siguientes pruebas:

- Test de organización sensorial.

- Límites de estabilidad.

- Test de adaptación.

- Desplazamiento máximo voluntario o control rítmico del peso.

\section{TEST DE ORGANIZACIÓN SENSORIAL}

La prueba de organización somatosensorial (SOT) nos permite analizar la aportación individual y combinada que cada sistema tiene en el mantenimiento del equilibrio.

Como se ha comentado previamente, el posturógrafo consta de una plataforma y un entorno móviles. Ambos se pueden mover, controlados por el ordenador, alrededor de un eje alineado con la articulación del tobillo. De este modo se pueden asociar las oscilaciones del cuerpo del paciente, con los movimientos de la plataforma y el entorno, de tal forma que estos se desplazarán en el mismo grado y dirección. Con ello se puede anular en gran medida, la información somatosensorial aportada por los receptores localizados en el tobillo (informan de las variaciones en el ángulo tibio tarsal) y se aporta una información visual errónea que indica que no hay movimiento, cuando realmente sí lo hay. Gracias a estas modificaciones en las aferencias de los sistemas encargados del mantenimiento del equilibrio podemos evaluar la contribución relativa de los sistemas visual y somatosensorial, y la habilidad para adaptarse a situaciones sensoriales conflictivas en condiciones de prueba referenciada a las oscilaciones corporales (sway-referenced) [5].

El estudio se ejecuta colocando al paciente sobre la plataforma y frente al entorno, se le pide que mantenga el equilibrio en seis condiciones diferentes en las que se realiza una distorsión selectiva de la información sensorial (Tabla1), haciendo tres intentos, de veinte segundos de duración, en cada una de ellas [7].

ANÁLISIS Y SIGNIFICADO CLÍNICO DE LOS RESULTADOS

GRADO DE ESTABILIDAD O PORCENTAJE DE EQUILIBRIO (Equilibrium score. Es un porcentaje que se obtiene de comparar el ángulo de desplazamiento anteroposterior máximo, con el esperado en una persona sana de su mismo sexo, edad y altura, para cada intento de cada condición. El resultado es un valor de 0 a 100, en el que 0 es la caída o el equilibrio peor y 100 el balanceo mínimo. A continuación, se establece un valor global, porcentual a lo normal, que pretende ser un dato representativo de la capacidad que tiene el un paciente para mantener el equilibrio (composite) [8]. Todos estos valores se representan en un diagrama de barras para que sea más sencilla la interpretación (Figura 2).

Se adjunta una tabla con el valor compuesto del equilibrio, a partir del cual se considera un resultado normal, ajustado por rango de edad (Tabla 2) [9].

Tabla 1. Condiciones de estudio en el test de organización somatosensorial.

\begin{tabular}{|l|l|}
\hline Condición 1 & $\begin{array}{l}\text { Ojos abiertos, entorno visual fijo y } \\
\text { plataforma fija. }\end{array}$ \\
\hline Condición 2 & Ojos cerrados y plataforma fija. \\
\hline Condición 3 & $\begin{array}{l}\text { Ojos abiertos, entorno visual móvil } \\
\text { (referenciado) y plataforma fija. }\end{array}$ \\
\hline Condición 4 & $\begin{array}{l}\text { Ojos abiertos, entorno visual fijo y } \\
\text { plataforma móvil (referenciada). }\end{array}$ \\
\hline Condición 5 & $\begin{array}{l}\text { Ojos cerrados y plataforma móvil } \\
\text { (referenciada). }\end{array}$ \\
\hline Condición 6 & $\begin{array}{l}\text { Ojos abiertos, entorno móvil } \\
\text { (referenciado) y plataforma móvil } \\
\text { (referenciado). }\end{array}$ \\
\hline
\end{tabular}

ANÁLISIS SENSORIAL. Para determinar cuál es el patrón o patrones sensoriales responsables de la alteración del equilibrio y el sistema sensorial en el que se apoya principalmente el control corporal, se realizan una serie de comparaciones y ratios de las condiciones sensoriales:

- Cociente somatosensorial. Compara la condición 2 con la condición 1 . Es en realidad el registro de la prueba de Romberg. El paciente al no tener información visual utiliza para equilibrarse el sistema somatosensorial. Un valor bajo, indica que el paciente es menos estable con los ojos cerrados lo que implica una alteración en la integración de la información somatosensorial y se denomina patrón de déficit somatosensorial (Figura 3).

- Cociente visual. Compara la condición 4 con la condición 1. Al disminuir la información somatosensorial (plataforma móvil referenciada a la oscilación corporal), el paciente utiliza para equilibrarse la 
información visual. La alteración aislada en la condición 4 determina un patrón de déficit visual.

- Cociente vestibular. Compara la condición 5 y la condición 1 . Al carecer de información somatosensorial y visual, el paciente utiliza para equilibrarse la información vestibular. Cuando existe alteración en la condición 5 o en la 5 y en la 6 , existe un patrón de déficit vestibular [10] (Figura 4).

- Cociente preferencia visual. Compara la suma de las condiciones 3 y 6 (en las que la información visual es errónea), con la de las condiciones 2 y 5 (donde se ha anulado la visión). Los pacientes con valores inferiores a los normales presentan un patrón de preferencia visual y presentan dificultad para mantener el equilibrio en circunstancias ambientales en que la información visual es errónea o imprecisa y no concuerda con la información correcta de origen vestibular o somatosensorial. Sin embargo, estos pacientes en ausencia de visión son capaces de mantener el equilibrio.

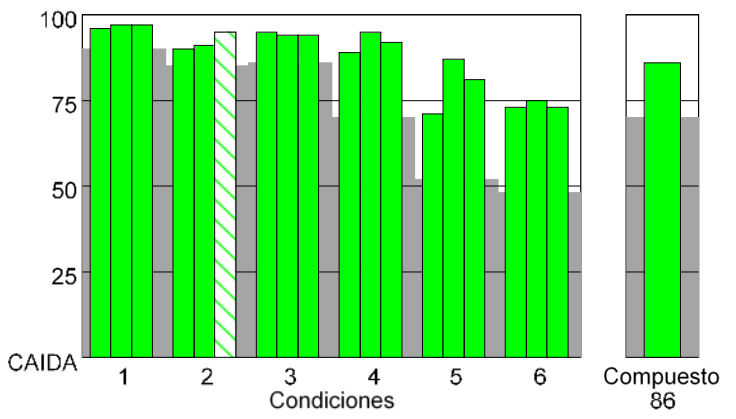

EQUILIBRIO

\section{Condiciones Prueba1Prueba2Prueba3}

\begin{tabular}{llll}
1 & 96 & 97 & 97 \\
2 & 90 & 91 & 95 \\
3 & 95 & 94 & 94 \\
4 & 89 & 95 & 92 \\
5 & 71 & 87 & 81 \\
6 & 73 & 75 & 73 \\
\multicolumn{4}{c}{ Compuesto $=86$}
\end{tabular}

Figura 2. Test de organización sensorial en paciente sano. Se presentan los resultados gráficos y numéricos. Como se aprecia los resultados están dentro de la normalidad, según su rango de edad sexo y altura (Sistema Neurocom® Versión 8.6.0 1989-2011).
Tabla 2: Tabla de valores normales del composite [9]

\begin{tabular}{|l|l|l|l|l|l|l|l|}
\hline AGE & $\begin{array}{l}\text { EQL- } \\
1\end{array}$ & $\begin{array}{l}\text { EQL- } \\
2\end{array}$ & $\begin{array}{l}\text { EQL- } \\
3\end{array}$ & $\begin{array}{l}\text { EQL- } \\
4\end{array}$ & $\begin{array}{l}\text { EQL- } \\
5\end{array}$ & $\begin{array}{l}\text { EQL- } \\
6\end{array}$ & COMP \\
\hline $3-4$ & 62.9 & 65.3 & 42.1 & 15.6 & 2.8 & 1.4 & 31.7 \\
\hline $5-6$ & 69.2 & 61.8 & 58.2 & 34.5 & 8.8 & 6.1 & 39.8 \\
\hline $7-8$ & 80.4 & 71.6 & 73.4 & 43.9 & 8.4 & 11.1 & 48.1 \\
\hline $9-10$ & 81.6 & 77.5 & 76.5 & 47.9 & 25.4 & 6.8 & 52.6 \\
\hline $\begin{array}{l}11- \\
13\end{array}$ & 86.6 & 85.7 & 82.2 & 52.2 & 21.8 & 23.3 & 58.6 \\
\hline $\begin{array}{l}14- \\
15\end{array}$ & 87.2 & 86.8 & 83.3 & 67.5 & 28.7 & 29.9 & 63.9 \\
\hline $\begin{array}{l}16- \\
59\end{array}$ & 90 & 85 & 86 & 70 & 52 & 48 & 70 \\
\hline $\begin{array}{l}60- \\
69\end{array}$ & 90 & 86 & 80 & 77 & 51 & 49 & 68 \\
\hline $\begin{array}{l}70- \\
79\end{array}$ & 70 & 63 & 82 & 69 & 45 & 27 & 64 \\
\hline
\end{tabular}

ALINEACIÓN DEL CENTRO DE GRAVEDAD (Figura 5). Es la representación gráfica del centro de gravedad sobre la plataforma al inicio de cada intento de cada condición y aporta información sobre la posible lateropulsión (anterior, posterior o lateral) del paciente. Esto nos va a permitir saber que hay que corregir en el control postural del paciente $y$, por tanto, es uno de datos a tener en cuenta en la programación de la rehabilitación vestibular [11].

ANÁLISIS DE LA ESTRATEGIA (Figura 6 y 7). Para poder interpretar y comprender esta información, vamos a explicar unos conceptos básicos. La estrategia es el tipo de movimiento realizado para mantener el centro de gravedad dentro de la base de sustentación. Existen tres tipos de estrategia: de tobillo, de cadera y de paso.

- Estrategia de tobillo. El movimiento del cuerpo para mantener el equilibrio se realiza sobre la articulación del tobillo. Se visualiza fácilmente al imaginarse el movimiento corporal sobre un balancín en dirección anteroposterior. El desplazamiento del centro de gravedad va ser lento y alejado de los límites de estabilidad. Este tipo de estrategia suele producirse cuando la superficie de soporte es estable o mayor que la de ambos pies.

- Estrategia de cadera. El movimiento del cuerpo se realiza sobre la articulación de la cadera. Es el balanceo sobre un balancín 
en dirección lateral. El centro de gravedad se desplaza rápidamente y se acerca a los límites de estabilidad. Se suele utilizar cuando la superficie es inestable y menos que la de los pies.

- Estrategia de paso. El desplazamiento del centro de gravedad va más allá de los límites de estabilidad lo que hace que se tenga que desplazar un pie para evitar la caída.
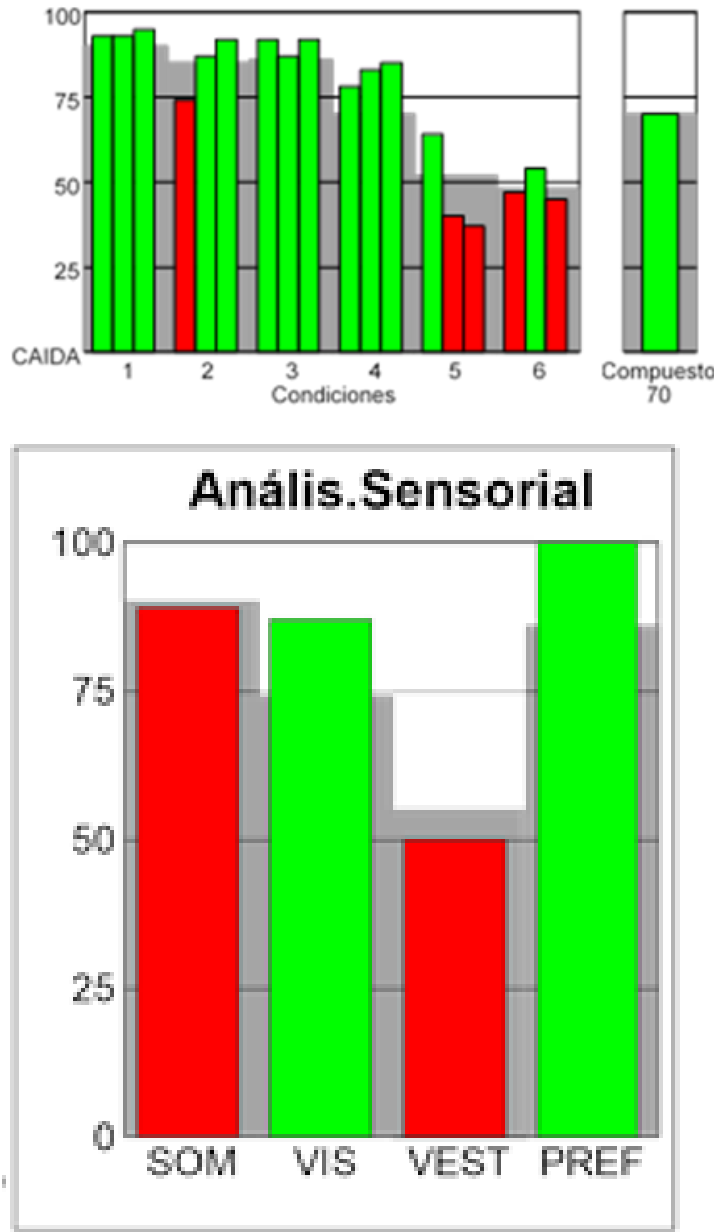

Figura 3. Combinación de patrones somatosensorial y vestibular en el Test de organización sensorial, en paciente con una neuronitis derecha (Sistema Neurocom $\AA$ Versión 8.6.0 1989-2011).
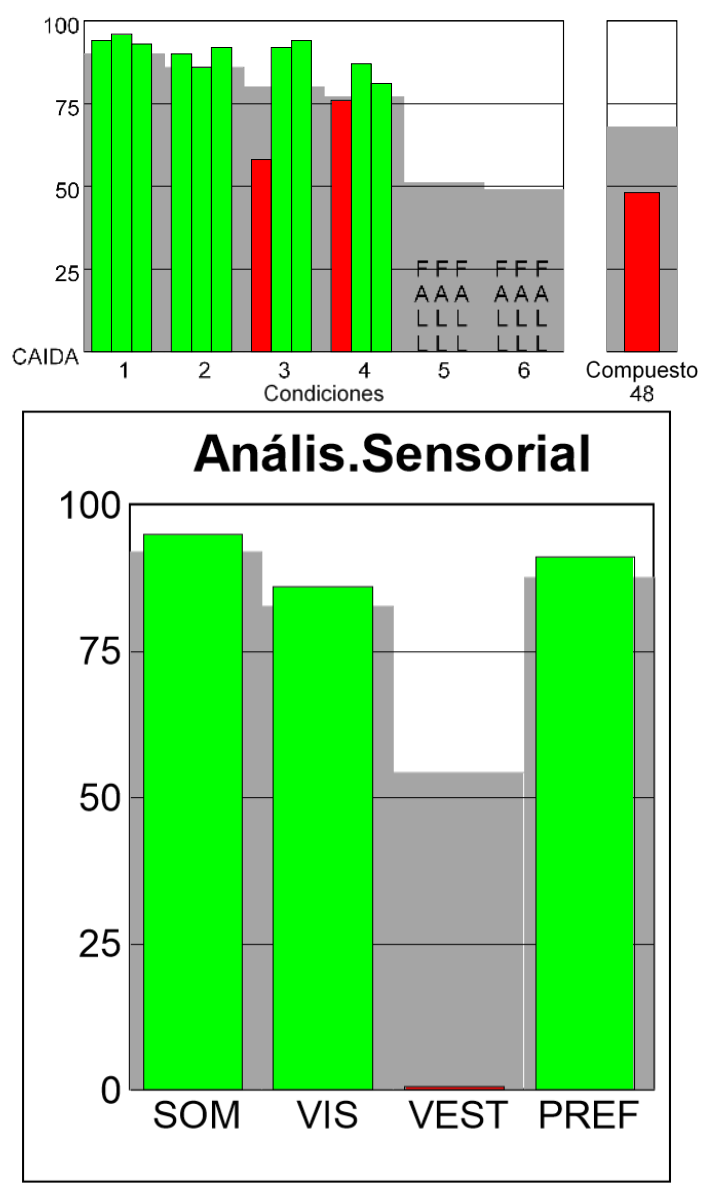

Figura 4. Test de organización somatosensorial en paciente con déficit vestibular bilateral. El resultado en las condiciones 5 y 6 es 0 , el resultado es un patrón vestibular (Sistema Neurocom® Versión 8.6.0 1989-2011).

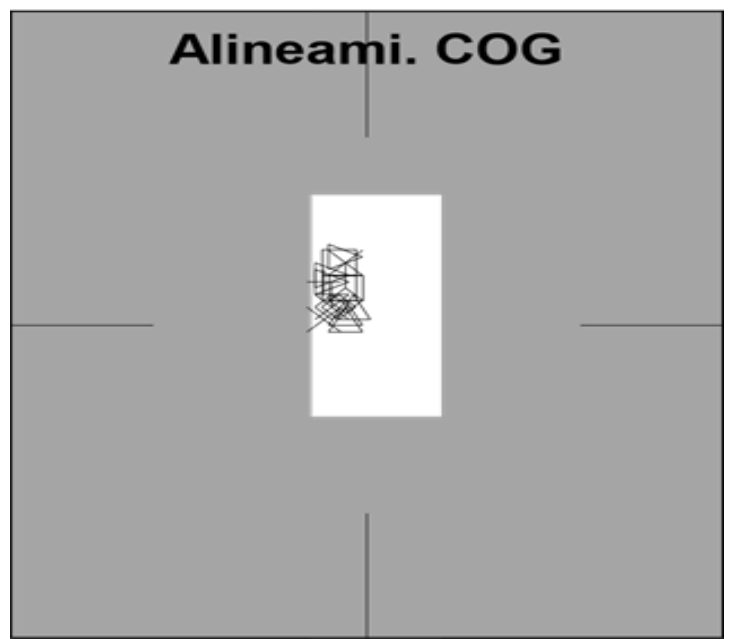

Figura 5. Representación del centro de gravedad (Sistema Neurocom® Versión 8.6.0 1989-2011). 


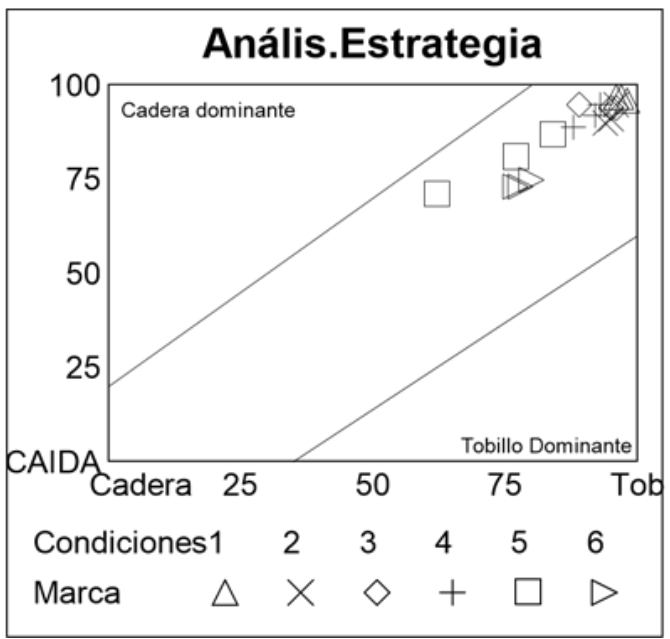

Figura 6. Diagrama de análisis de estrategia en un paciente con un patrón de normalidad (Sistema Neurocom ${ }^{\circledR}$ Versión 8.6.0 1989-2011).

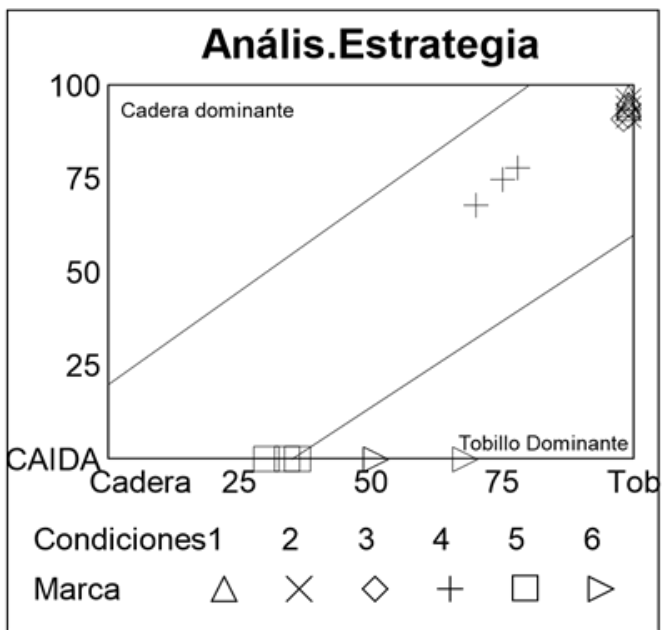

Figura 7. Diagrama de análisis de estrategia en un paciente con un patrón vestibular (Sistema Neurocom® Versión 8.6.0 1989-2011).

Una persona sin trastornos del equilibrio utiliza con mayor frecuencia la estrategia de tobillo para las superficies estables y a medida que la superficie se hace más irregular, va incorporando la estrategia de cadera en su movimiento [12].

En el análisis de la estrategia obtenemos un diagrama de porcentajes de estabilidad, de la cantidad de movimientos de tobillo o de cadera, que utiliza el paciente en cada condición sensorial analizada. El eje vertical representa la estabilidad o ausencia de balanceo, siendo el $100 \%$ la mayor estabilidad y 0 la caída. En el eje horizontal se representan la estrategia de tobillo hacia la derecha y la estrategia de cadera hacia la izquierda. Por lo que, si el paciente tiene buena estabilidad, los pacientes se encontrarán en la porción superior derecha de la gráfica.

PATRÓN AFISIOLÓGICO (Figura 8). Puede aparecer en pacientes simuladores, con trastornos somatomorfos, en la depresión y en trastornos de ansiedad; los cuales tienden a exagerar la incapacidad. Son pacientes que entran en la consulta por su propio pie o sin presentar un déficit postural evidente; y obtienen un porcentaje global del equilibrio muy disminuido, incluso realizan mal los tres intentos de la condición 1.

Cevette describe los siguientes criterios de patrón afisiológico:

- Una estrategia es afisiológica cuando el paciente se comporta posturalmente mejor en las condiciones más difíciles (4, 5 y 6$)$, con mayor conflicto sensorial, que en las más sencillas (1 y 2).

- Cuando existe una gran variabilidad entre los intentos de una misma condición, lo que conforma una respuesta errática. En cada condición se realizan tres intentos, lo normal es que la respuesta en cada uno de ellos sea idéntica o que incluso mejoré en cada repetición (adaptación).

- Cevette et al. definieron tres fórmulas, cada una de las cuales determina el patrón normal, afisiológico y vestibular. El patrón definitivo es el que obtiene valor numérico mayor [13].

Estos pacientes van a presentar unos patrones de oscilación circular o lateral excesivos, sin caída o previos a las mismas. También grandes oscilaciones con regularidad, bien controladas, que no aumentan al complicarse la prueba. Además, si repetimos la prueba o cambiamos de orden la realización de las condiciones o estímulos vamos a obtener siempre resultados diferentes e irrepetibles.

\section{LÍMITES DE ESTABILIDAD:}

La CDP permite conocer los límites de estabilidad del paciente, es decir, el área en el que puede mover con seguridad su centro de presiones sin perder el equilibrio. Para ello se le pide que, viendo la representación de su centro de presiones en la pantalla situada frente a él, la desplace en 8 direcciones diferentes, que 
van a ir apareciendo mediante dianas en el monitor, siempre partiendo de la posición central. La prueba está normalizada a la altura y peso del sujeto que la realiza.

Esta prueba permite visualizar si el paciente ha alcanzado todos los objetivos, con la trayectoria empleada para ello y cuantificar la habilidad del paciente para desplazar voluntariamente su centro de gravedad, a través de los siguientes parámetros (Figura 9):

1. Tiempo de reacción: tiempo en segundos desde que se ilumina la diana y el inicio del desplazamiento. El aumento del tiempo de reacción se asocia a alteraciones del SNC, motoras o a la falta de colaboración.

2. Velocidad de movimiento: velocidad en grados/segundo del desplazamiento del centro de presiones. La disminución de la velocidad de movimiento aparece en alteraciones centrales como el Parkinson o que cursen con importante rigidez articular [14].

3. Punto de excursión inicial (EPE, Endpoint Excursion): distancia expresada en porcentaje de límites de estabilidad, recorrida en el primer intento de alcanzar la diana correspondiente.

4. Punto de excursión máxima (MXE): distancia máxima alcanzada (en \% de límites de estabilidad). EPE y MXE son dos parámetros muy útiles para planificar la rehabilitación vestibular.

5. Control direccional: comparación entre la cantidad de movimiento hacia el objetivo y en dirección contraria.

Se han observado pequeñas diferencias según el sexo, en hombres existe menor tiempo de reacción y mayor velocidad, sin que existan diferencias ni en el recorrido ni el control direccional, lo que implica que una mayor celeridad no implica un mejor control del equilibrio [15]. El conocimiento de estos límites aporta información de la capacidad del sujeto de desplazarse de forma segura, siendo de gran ayuda en la planificación del tratamiento rehabilitador (Figura 10), que tiene como fin aumentar los límites de estabilidad trabajando mediante feedback [11].

\section{TEST DE ADAPTACIÓN:}

Permite valorar la capacidad de reacción del paciente ante movimientos bruscos e inesperados. La prueba se realiza mediante rápidas rotaciones de la plataforma (5 hacia adelante y 5 hacia atrás) que provocan respuestas automáticas para contrarrestar la tendencia a desplazarse.

Se analiza la magnitud del balanceo que realiza el sujeto durante los 2,5 s siguientes a la inclinación súbita. Una amplitud que va disminuyendo con cada intento es un patrón normal y representa una buena capacidad de adaptación (Figura 11).

Un resultado patológico, que puede aparecer en múltiples alteraciones (hipofunción vestibular no compensada, centrales, de la articulación del tobillo) se relaciona con una mayor frecuencia de caídas y mayor dificultad para deambular por superficies irregulares o inestables.

También es de utilidad para determinar un patrón afisiológico si aparecen respuestas exageradas pasando el primer segundo después del estímulo, ya que este periodo pertenece al control voluntario de la estabilidad [16]. 


\section{Test Organización Sensorial}
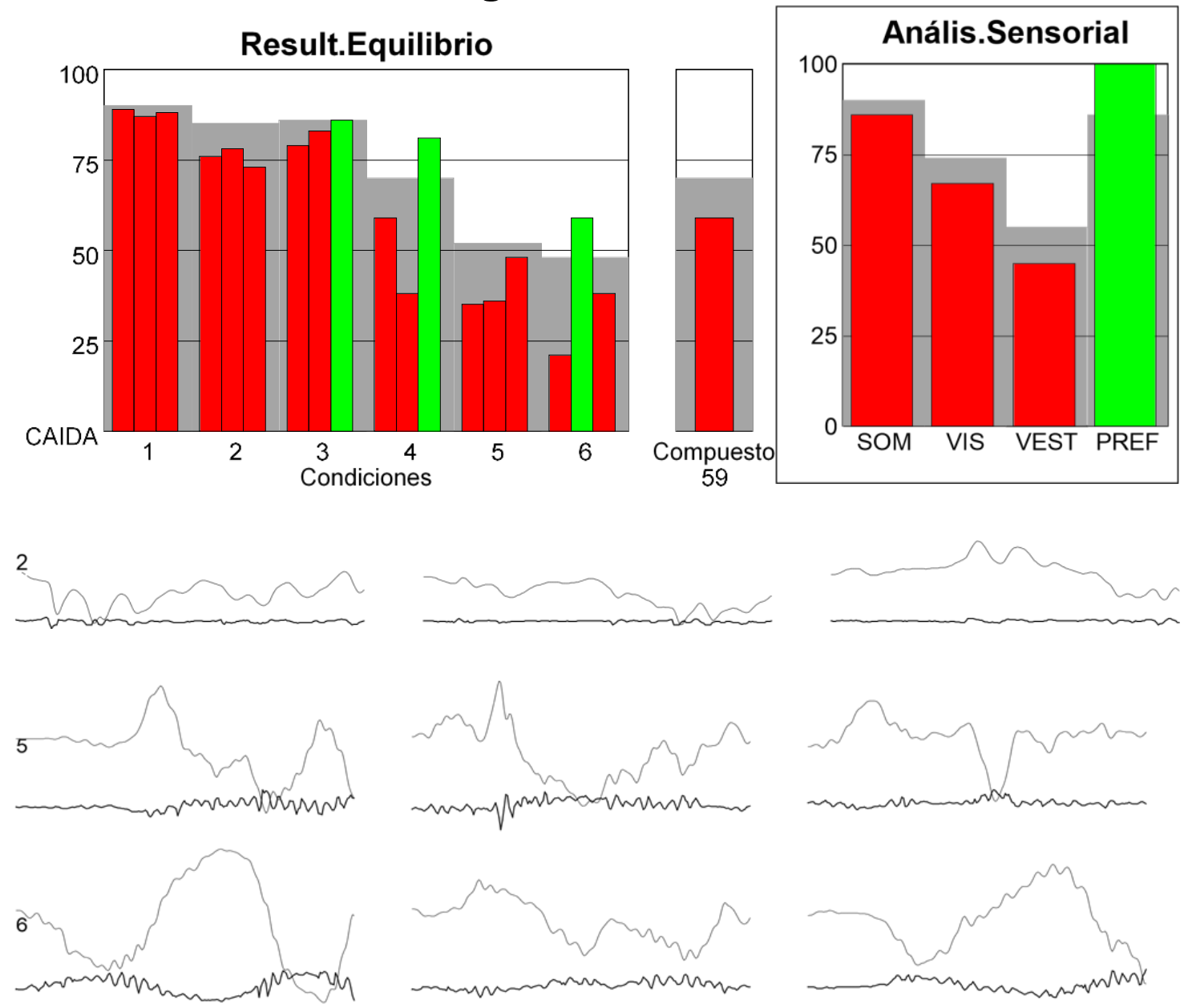

$=5$ degrees; $16 \mathrm{~kg}$ force

20 segund.

Figura 8. Patrón afisiológico. En el paciente se comporta posturalmente mejor en las condiciones más complejas (5 y 6$)$ que en las más sencillas ( 1 y 2 ). Se puede apreciar que no hay una gran variación de las oscilaciones entre la condición 2 y las condiciones 5 y6 (Sistema Neurocom® Versión 8.6.0 1989-2011). 


\section{Límites Estabilidad}

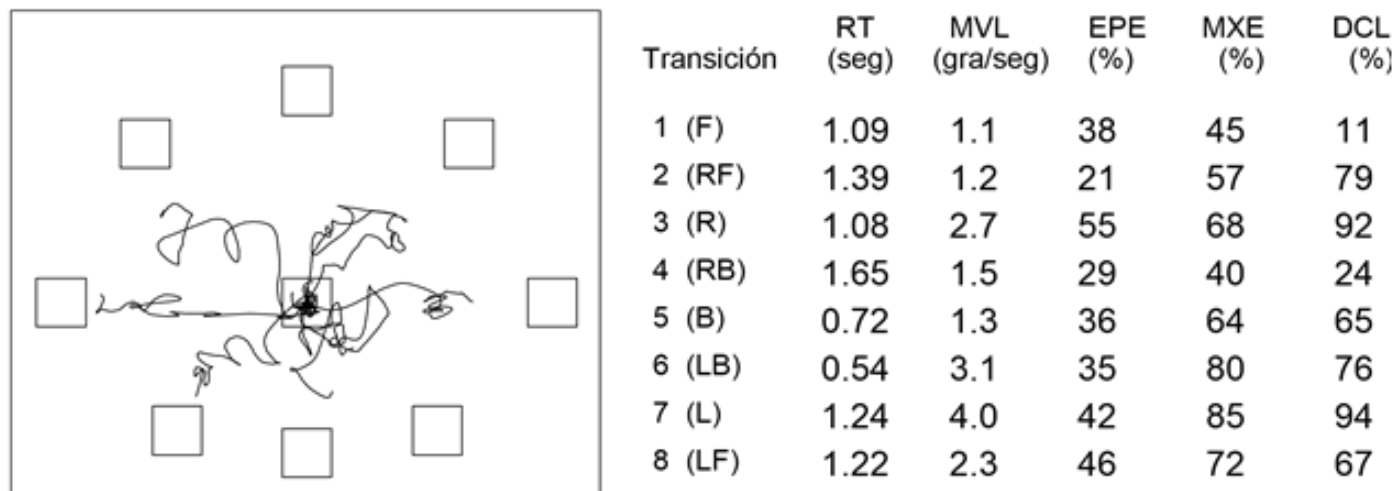

$100 \%$ LOS
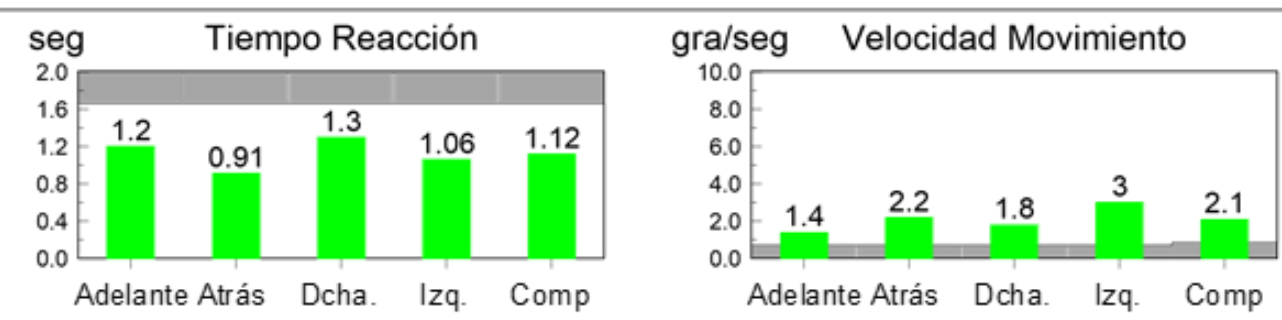

\% Punto de Excursión final máxima

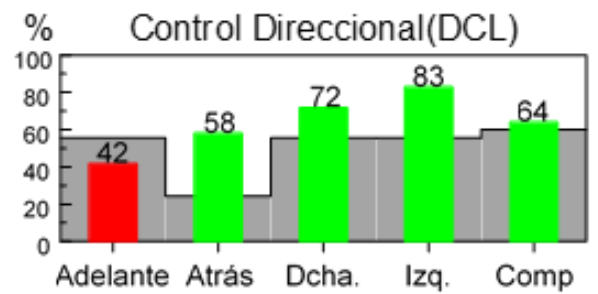

Figura 9. Límites de estabilidad en paciente con un déficit vestibular izquierdo (Sistema Neurocom® Versión 8.6.0 19892011).
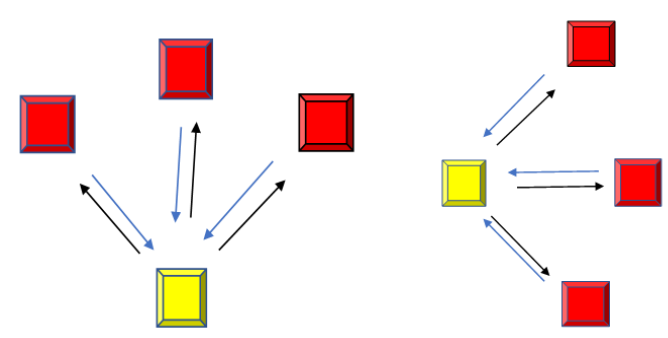

Figura 10. Ejercicios de rehabilitación diseñados para mejorar los límites de estabilidad con el posturógrafo.

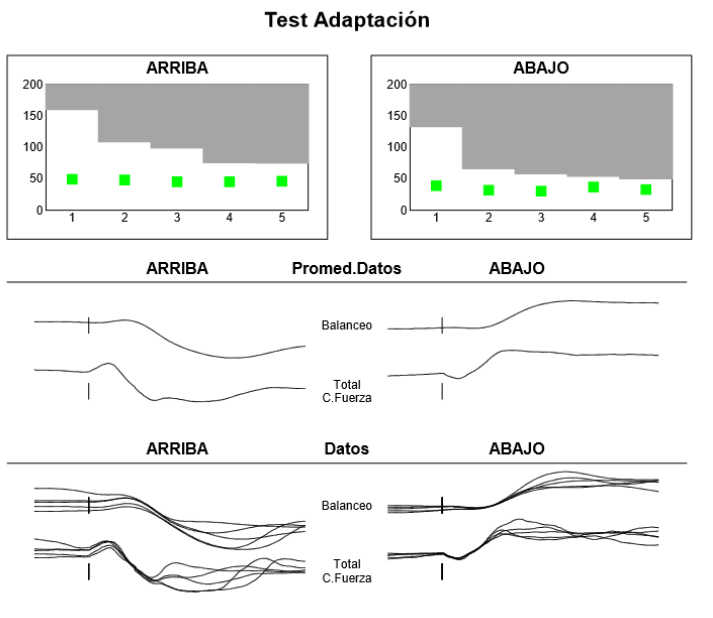

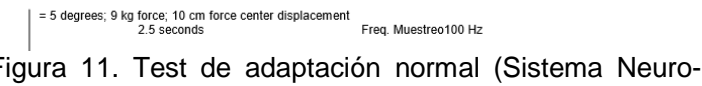
com® Versión 8.6.0 1989-2011). 
CONTROL RÍTMICO Y DIRECCIONAL:

Permite conocer la capacidad del paciente para realizar desplazamientos rítmicos de su centro de gravedad. La prueba consiste en que el sujeto debe seguir con la representación de su centro de presiones, una diana que se moviliza en el plano anteroposterior y lateral, a diferentes velocidades (Figura 12).

Se determinan dos parámetros: la velocidad sobre el eje y el control direccional.

Un resultado patológico puede implicar dificultad para realizar actividades con variación de velocidad o cambios de dirección (cruzar la calle, subir escaleras...).

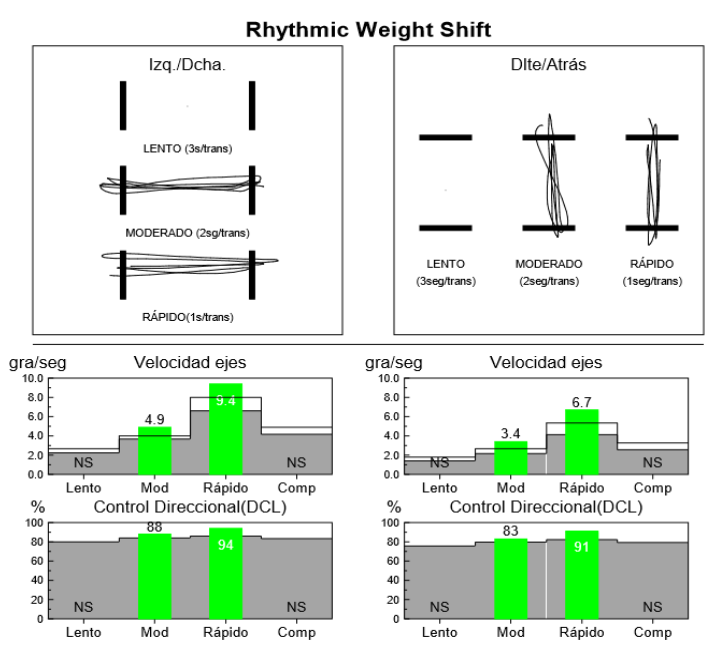

Figura 12. Test de control del ritmo (Sistema Neurocom® Versión 8.6.0@ 1989-2011).

\section{CONCLUSIONES}

La posturografía dinámica computarizada es una prueba diagnóstica y terapéutica, que no permite llegar a un diagnóstico etiológico, ya que no existen patrones patognomónicos para cada patología, pero sí permite cuantificar de forma objetiva y reproducible la estabilidad del paciente. Y aporta información relevante para planificar el tratamiento rehabilitador, así como para monitorizar su evolución.

Es importante destacar la utilidad medicolegal de la CDP, tanto para la valoración objetiva del efecto de las alteraciones del equilibrio sobre las actividades de la vida cotidiana del sujeto, como para realizar un informe médico en pacientes sospechosos de conductas simuladoras, gracias a la descripción de un patrón afisiológico.
Hay algunos factores a tener en cuenta para evitar errores en la realización e interpretación de la prueba como son: las condiciones físicas, el nivel intelectual y de aprendizaje, y la colaboración y la motivación del paciente al realizar la prueba o si está en tratamiento con algún tipo de medicación sedante. En ocasiones, es conveniente parar para realizar una explicación más detallada o descansar; incluso a veces, repetir la prueba en otro momento.

\section{BIBLIOGRAFÍA}

1. Subcommitte of the American Academy of Neurology. Assessment: Posturography. Neurology. 1993; 43(6):1261-4.

2. Furman JM. Role of posturography in themanagement of vestibular patients. Otolaryngol Head Neck Surg. 1995; 112 : 8-15.

3. Pérez Fernández N. Atlas de pruebas vestibulares para especialistas en otorrinolaringología. Barcelona: Profármaco; 2009.

4. NeuroCom International. NeuroCom System Operators Manual. 8 th ed. Clackamas: NeuroCom International Inc; 2001.

5. Nashner LM, Peters JF: Dynamic posturography in the diagnosis and management of dizziness and balance disorders. En: Arenberg IK, Smith DB, eds. Neurologic Clinics: Diagnostic Neurotology. Philadelphia: WB Saunders Company; 1990: 331-349.

6. Black FO. What can posturography tell us about vestibular function? Ann N Y Acad Sci. 2001; 942:446-64.

7. Shepard NT, Janky K. Background and Technique of computarized dynamic posturography. En: Jacobson GP, Shepard NT, eds. Balance function assessment and management. San Diego: Plural Publishing Inc; 2008:339-357.

8. Barona R, Quinzá V, Martín E. Posturografía ¿algo se mueve? [Internet]. Valencia: Instituto biomecánica de Valencia; 2003. [actualizado feb 2003; citado nov 2017]. Disponible en: http://www.ibv.org/publicaciones/catalogodepublicaciones/posturografia-algose-mueve. 
9. Nasher LM: Computerized dynamic posturography clinical applications. En: Jacobson GP, Newman CW, Kartush JM, eds. Handbook of Balance Function Testing. St. Louis: Mosby Year Book, 1997: 308-334.

10. Doménech-Vadillo E, Montes-Jovella L, Rey-Martínez J, Pérez-Fernández $\mathrm{N}$. Los patrones normal y vestibular en la posturografía dinámica de pacientes con enfermedad de Menière. Acta Otorrinolaringol Esp. 2010; 61: 34-40.

11. Santos Pérez S, Soto Varela A, Rossi lzquierdo M, Elhendi W, Labella Caballero T. Tratamiento rehabilitador de los trastornos del equilibrio. An Otorrinolaringol Ibero Am. 2007; 34 (5):465-84.

12. Peydro de Moya MF, Baydal Bertomeu, Vivas Broseta MJ. Evaluación y rehabilitación del equilibrio mediante posturografía. Rehabilitacion. 2005; 39 (6): 31523.
13. Cevette MJ, Puetz B, Marion MS, Wertz $\mathrm{ML}$, Muenter MD. Aphysiologic performance on dynamic posturography. Otolaryngology Head and Neck surgery. 1995; 112:676-88.

14. Rossi M, Soto A, Santos S, Sesar A, Labella $T$. A prospective study of alterations in balance among patients with Parkinson's disease. Protocol of the postural evaluation. Eur Neurol. 2009; 61 (3):171-6.

15. Faraldo-García A, Santos-Pérez S, Labella-Caballero T, Soto-Varela $A$. Influencia del sexo en el test de organización sensorial y los límites de estabilidad en sujetos sanos. Acta Otorrinolaringol Esp. 2011 Sep-Oct; 62(5):333-8.

16. Rey-Martínez J, Rama-López J, PérezFernández N. Exploración posturográfica de pacientes simuladores. Acta Otorrinolaringol Esp 2007; 58: 202-7. 